\title{
A note on non-smooth programming problems
}

\author{
Mohammad Mehdi Mazarei*, Ali Vahidian Kamyad, Ali Asghar Behroozpoor \\ Department of Applied Mathematics, Faculty of Mathematical Sciences, Ferdowsi University of Mashhad, International Campus, \\ Mashhad, Iran
}

\section{A R T I C L E I N F O}

\section{Article history:}

Received 15 June 2016

Received in revised form

29 July 2016

Accepted 30 July 2016

\section{Keywords:}

Nonlinear programming

Linear programming

Piecewise linearization

Non-smooth function

\begin{abstract}
A B S T R A C T
In this paper, we introduce a new approach to obtain a novel numerical solution of nonlinear programming problems (NLP) which the objective function (functions) or constraint function (functions) are non-smooth ones. This technique is based on a new piecewise linearization approach. In fact, we transfer the nonlinear programming problem (NLP) to a variational problem that would reduce the new approximated problem to a linear programming problem (LP). Then, the approximated solution of the original problem would be obtained by the LP problem. Finally, numerical examples are given to show the efficiency of the proposed approach.
\end{abstract}

(C) 2016 The Authors. Published by IASE. This is an open access article under the CC BY-NC-ND license (http://creativecommons.org/licenses/by-nc-nd/4.0/).

\section{Introduction}

A wide range of problems arising in practical applications can be formulated as nonlinear programs (NLPs). This includes chemical engineering (Grossmann and Sargent, 1979; Corsano et al., 2011), water network problems (Misener and Floudas, 2012), gas (Bragalli et al., 2006), energy (Murray and Shanbhag, 2006), time-loss in the paper industry (Harjunkoski et al., 1999), concrete structure design (Guerra et al., 2011), load-bearing thermal insulation systems (Abhishek et al., 2010), medical applications (Pardalos et al., 2004) and so on. There are some methods and algorithms available in the literature for the case in which the objective and constraint functions are convex and differentiable, e.g. the optimal point can be obtained using Karush-Kuhn-Tucker conditions. Furthermore, the penalty and barrier methods can be used for constraint optimization problems. Although, some other methods based on linear programming exist, such as the method of approximate programming (Griffith and Stewart, 1961; Kamyad et al., 2005). After Han proved local and global convergence of SQP methods in (Han, 1976; Han, 1977), a large amount of research papers have been produced on SQP-based techniques. However, all of the aforementioned techniques are for differentiable (smooth) or convex problems and some optimality

\footnotetext{
* Corresponding Author.

Email Address: mazarei mehdi@yahoo.com (M. Mazarei) http://dx.doi.org/10.21833/ijaas.2016.07.014

2313-626X/@ 2016 The Authors. Published by IASE.

This is an open access article under the CC BY-NC-ND license

(http://creativecommons.org/licenses/by-nc-nd/4.0/)
}

conditions are necessary such as continuity, convexity and differentiability. But, many important practical problems are naturally modeled as nonsmooth NLPs and these methods are inadequate to solve them.

The non-smooth NLPs would be useful in much application of sciences. Some investigations have been done in non-smooth programming problems (Rockafellar, 1994; Nesterov, 2005). However, these techniques are not efficient for non-convex nonsmooth optimization problems. It is worthwhile to mention that many well-studied optimization problems can also be naturally viewed as nonconvex and non-smooth NLPs. In this study, we have proposed a new technique to solve nonlinear nonsmooth programming problems.

The rest of this paper is organized as follows. In next section, we introduce a technique to find the best piecewise linearization of nonlinear functions. In section 3, we explain the equivalency of non-linear and linear programming problem. In Section 4, we illustrate some numerical examples to demonstrate the efficiency and accuracy of the proposed approach.

\section{A new piecewise linearization of nonlinear function}

The linearization of nonlinear systems is an efficient tool for finding approximate solutions and treatment analysis of these systems.

Remark 2.1: Since, every non-smooth function is a nonlinear function, we consider nonlinear functions.

Let $F: A \subseteq R^{n} \rightarrow R$ be a nonlinear function. We suppose that $x \in A \subseteq R^{n}$ and the subset $A$ is 
compact. Our aim is to approximate the nonlinear function $F$ by a piecewise linear function as follows (Eq. 1):

$$
\begin{aligned}
& F_{N}(x)= \\
& \sum_{i=1}^{N}\left(a_{i 0}+a_{i 1} x_{1}+a_{i 2} x_{2}+\cdots+a_{i n} x_{n}\right) X_{A_{i}}(x) \\
& a_{i j} \in R ; i=1,2, \ldots, N
\end{aligned}
$$

where, $A_{i}$ is $i$ th subset in partitioning of $A$ as $P_{N}=\left\{A_{1}, A_{2}, \ldots, A_{N}\right\}$. As we know, this partitioning has the bellow properties:

1) $\forall i, j=1,2, \ldots, N ; A_{i} \cap A_{i}=\emptyset ; A_{i} \in R^{n}, A_{i} \neq \emptyset$

2) $A=\bigcup_{i=1}^{N} A_{i}$

also, $A_{i}$ is Lebesgue measurable set and $X_{A_{i}}(x)$ be the characteristic function on $A_{i}$ defined as follows (Eq. 2):

$$
\begin{aligned}
& X_{A_{i}}(x)= \begin{cases}1, & x \in A_{i} \\
0, & x \notin A_{i}\end{cases} \\
& L_{1}(A)=\left\{f: A \rightarrow R\left|\int_{A}\right| f \mid d x<\infty\right\} \\
& L_{2}(A)=\left\{f:\left.A \rightarrow R\left|\int_{A}\right| f\right|^{2} d x<\infty\right\} .
\end{aligned}
$$

Now, let and them. As we know $L_{2}(A)$ is a Hilbert space of $A$ with the following inner product (Eqs. 3 and 4):

$$
\begin{aligned}
& \langle f, g\rangle=\left(\int_{A} f(x) g(x) d x\right)^{1 / 2} ; f, g \in L_{2}(A) \\
& \text { and } \\
& \|f\|_{2}=\left(\int_{A}|f|^{2} d x\right)^{1 / 2}
\end{aligned}
$$

Definition 2.1: We define $S_{N}(A) ;(N \in N)$ be the set of all $F_{N} \in L_{1}(A)$ of the form (1).

Lemma 2.1: For $0<\mathrm{P}<\mathrm{q}$ we have $L_{P} \subset L_{q}$.

So, we have $L_{1} \subset L_{2}$. We use the norm-1 in the rest of paper.

Definition 2.2: If $F: R^{n} \rightarrow R$ is a nonlinear function and $F_{N} \in S_{N}(A)$, we define $\left\|F-F_{N}\right\|_{1}$ as follows (Eq. 5):

$$
\left\|F-F_{N}\right\|_{1}=\int_{A}\left|F-F_{N}\right| d x
$$

Lemma 2.2: The subset $S_{N}(A)$ is dens on $L_{1}(A)$.

Proof: Suppose that $\mathrm{F}$ is a nonlinear function that,

$F: A \subseteq R^{n} \rightarrow R$

$\forall \varepsilon>0 \exists N(\varepsilon) \in N, \exists F_{N}(.) \in S_{N}(A)$

$\left\|F-F_{N}\right\|_{1}<\varepsilon$.

Definition 2.3: We call $F^{*} \in S_{N}(A)$ the best piecewise linear approximation of $F$ if for any $F_{N} \in S_{N}(A)$ we have,

$$
\left\|F-F^{*}\right\|_{1} \leq\left\|F-F_{N}\right\|_{1}
$$

therefore, $F^{*}$ is the optimal solution of the following optimization problem (Eq. 6).

$$
\begin{aligned}
& M i n\left\|F-F_{N}\right\|_{1} \\
& F \in S_{N}(A)
\end{aligned}
$$

Obviously, $\quad$ because $0 \leq\left\|F-F_{N}\right\|_{1}$, the optimization problem (6) has optimal solution.

To clarify our approach, first we consider a nonlinear function $F: R \rightarrow R$. Second, we explain this approach for a nonlinear function $F: R^{n} \rightarrow R$.

(i) Consider the optimization problem

$$
\begin{aligned}
& \operatorname{Min}\left\|F-F_{N}\right\|_{1} \\
& f \in S_{N}(A)
\end{aligned}
$$

where, $F: A \subseteq R \rightarrow R$ is a nonlinear function and $A=[a, b]$. As we know $[a, b]$ can be replaced by $[0,1]$.

Now, we decompose interval $[0,1]$ to $N$ subintervals $\left[\frac{i-1}{N}, \frac{i}{N}\right] ; 1,2, \ldots, N$ (Fig. 1). Since, $F_{N} \in$ $S_{N}(A)$, we have (Eq. 7)

$$
\operatorname{Min} \int_{0}^{1}\left|F(x)-\sum_{i=1}^{N}\left(a_{i}+b_{i} x\right) x_{\left[\frac{i-1}{N}, \frac{i}{N}\right]}(x)\right| d x
$$

Our objective function is a functional. Now, we reduce this functional to a summation as follows (Eq. 8):

$$
\begin{aligned}
& \int_{0}^{1}\left|F(x)-\sum_{i=1}^{N}\left(a_{i}+b_{i} x\right) x_{\left[\frac{i-1}{N}, \frac{i}{N}\right]}(x)\right| d x \cong \\
& \frac{1}{N} \sum_{i=1}^{N}\left|F\left(x_{i}\right)-\left(a_{i}+b_{i} x\right)\right|
\end{aligned}
$$

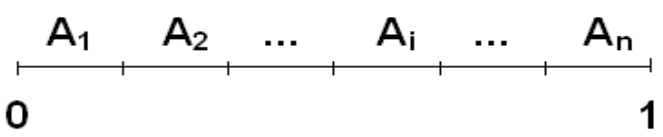

Fig. 1: Partitioning $A$ to subintervals $A_{i}$.

so, the optimization problem (8) is as follows (Eq. 9):

$$
\begin{aligned}
& \operatorname{Min} \sum_{i=1}^{N}\left|r_{i}+s_{i}\right| \\
& r_{i}-s_{i}=F\left(x_{i}\right)-\left(a_{i}+b_{i} x_{i}\right) \\
& 0 \leq r_{i}, s_{i} ; i=1,2, \ldots, N
\end{aligned}
$$

But, the optimization problem (9) is a nonlinear programming problem. We reduce this problem to a linear programming problem by relation $\left|r_{i}-s_{i}\right|=$ $r_{i}+s_{i}$. So, our optimization problem will be as the following linear programming problem (Eq. 10):

$$
\begin{aligned}
& \operatorname{Min} \frac{1}{N} \sum_{i=1}^{N} r_{i}+s_{i} \\
& \text { s.t } \\
& r_{i}-s_{i}=F\left(x_{i}\right)-\left(a_{i}+b_{i} x_{i}\right) \\
& 0 \leq r_{i}, s_{i} ; i=1,2, \ldots, N
\end{aligned}
$$

(ii) Second, we consider a nonlinear function $F: A \subseteq R^{n} \rightarrow R$ then the optimization problem would be as follows:

$$
\begin{aligned}
& \operatorname{Min} \int_{A} \mid F\left(x_{1}, x_{2}, \ldots, x_{n}\right)-\sum_{i=1}^{N}\left(a_{i 0}+a_{i 1} x_{1}+\right. \\
& \left.a_{i 2} x_{2}+\cdots+a_{i n} x_{n}\right) X_{A_{i}}(x) \mid d x
\end{aligned}
$$

As, we explained in section (i) this optimization problem will be reduced to a linear programming problem as follow (Eq. 11): 


$$
\begin{aligned}
& \operatorname{Min} \sum_{i=1}^{N} r_{i}+s_{i} \\
& \text { s.t } \\
& r_{i}-s_{i}=F\left(x_{1}, x_{2}, \ldots, x_{n}\right)-\left(a_{i 0}+a_{i 1} x_{1}+a_{i 2} x_{2}+\right. \\
& \left.\quad \cdots+a_{i n} x_{n}\right) \\
& 0 \leq r_{i}, s_{i} ; i=1,2, \ldots, N \\
& N=m_{1}, m_{2}, \ldots, m_{n}
\end{aligned}
$$

Where, $m_{1}, m_{2}, \ldots, m_{n}$ are the numbers of subintervals on axises $x_{1}, x_{2}, \ldots, x_{n}$, respectively.

\section{Nonlinear programming problem}

Now, we consider the nonlinear programming problem as follows:

$$
\begin{aligned}
& \operatorname{Min} F\left(x_{1}, x_{2}, \ldots, x_{n}\right) \\
& \text { s.t } \\
& G_{i}\left(x_{1}, x_{2}, \ldots, x_{n}\right) \geq 0 ; i=1,2, \ldots, m \\
& G_{k}\left(x_{1}, x_{2}, \ldots, x_{n}\right)=0 ; k=m+1, m+2, \ldots, l
\end{aligned}
$$

where, the objective function $F$ or constraint functions $G_{i}, G_{k}$ are nonlinear functions. According to the previous section, we replace objective and constraint functions by the best piecewise linear functions of form (1). Therefore, the nonlinear programming problem (12) is approximately equal to a linear programming problem as follows:

$$
\begin{aligned}
& \operatorname{Min} \sum_{i=1}^{N}\left\{a_{i 0}+a_{i 1} x+a_{i 2} y\right\} X_{A_{i}}(x, y) \\
& \text { s.t } \\
& \sum_{i=1}^{N}\left\{b_{i 0}+b_{i 1} x+b_{i 2} y\right\} X_{A_{i}}(x, y) \geq 0 ; i=1,2, \ldots, n \\
& \sum_{i=1}^{N}\left\{c_{i 0}+c_{i 1} x+c_{i 2} y\right\} X_{A_{i}}(x, y)=0 ; k=m+ \\
& 1, m+2, \ldots, l
\end{aligned}
$$

Now, according to lemma1, we can approximate objective function $F$ and constraint functions $G_{i}$ and $G_{k}$ arbitrarily. Then, the objective function and feasible region in nonlinear programming problem (12) is approximated to the objective function and feasible region in linear programming problem (13). This lead to the optimal solution of linear programming problem (12) is the approximated optimal solution of problem (13). This is clear we can improve the accuracy of approximated optimal solution by increasing $\mathrm{N}$.

\section{Numerical examples}

In this section, we show the efficiency of new technique by some examples.

Example 1: Consider following nonlinear smooth programming problem:

$$
\begin{aligned}
& \text { Min } e^{x} \\
& \text { s.t } \\
& \sin (x)-x \leq 1 \\
& 1 \leq x \leq 3
\end{aligned}
$$

We may convert interval $[1,3]$ to $[0,1]$. For this purpose, we may define bijective function $H(x)$ as follows:

$$
\begin{aligned}
& H:[1,3] \rightarrow[0,1] \\
& x \rightarrow \frac{x-1}{2} \\
& \text { Now, we have: } \\
& \text { Min } e^{(2 x+1)} \\
& \text { s.t } \\
& \sin (2 x+1)-2 x \leq 2 \\
& 0 \leq x \leq 1
\end{aligned}
$$

The optimal solution is $x^{*}=3$. We have used the piecewise linearization of objective function and constraint functions to approximate non-smooth programming problem (15) to a linear programming problem. The optimal solution of approximated linear programming problem for $n=10,20,50,100$ has been showed in Table 1.

Table 1: The approximate optimal point of example 1

\begin{tabular}{|c|c|}
\hline $\mathrm{n}$ & Approximate optimal solution \\
\hline 10 & 2.9020 \\
\hline 20 & 2.9813 \\
\hline 50 & 2.9900 \\
\hline 100 & 2.9950 \\
\hline
\end{tabular}

Example 2: Consider following nonlinear nonsmooth programming problem:

$$
\begin{aligned}
& \text { Min } e^{|2 x-1|} \\
& \text { s.t } \\
& \sin (x) \leq 1 \\
& 0 \leq x \leq 1
\end{aligned}
$$

In this example, the objective function is nonsmooth function. The exact optimal solution of nonlinear non-smooth programming problem (16) is $x^{*}=1$.

We have used the piecewise linearization of objective function and constraint function to approximate non-smooth programming problem (16) to a linear programming problem (Figs. 2 and 3). The optimal solution of approximated linear programming problem for $n=10,20,50,100$ has been showed in Table 2 .

Table 2: The approximate optimal point of example 2

\begin{tabular}{|c|c|}
\hline$n$ & Approximate optimal solution \\
\hline 10 & 0.9471 \\
\hline 20 & 0.9631 \\
\hline 50 & 0.9802 \\
\hline 100 & 0.9943 \\
\hline
\end{tabular}

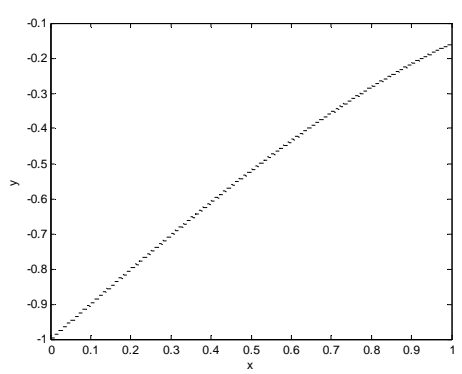

Fig. 2: The piecewise linearization of constraint function of example $2(n=100)$. 


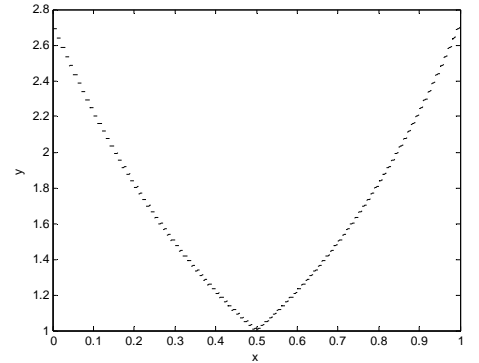

Fig. 3: The piecewise linearization of objective function of example $2(n=100)$

Example 3: Consider following nonlinear nonsmooth programming problem:

$$
\begin{aligned}
& \text { Min }\left|x-y^{3}\right| \\
& \text { s.t } \\
& y-x^{2} \geq 0 \\
& y+x-2 \geq 0 \\
& 0 \leq x, y
\end{aligned}
$$

In this example, the objective function is nonlinear non-smooth function. The exact optimal solution of nonlinear programming problem (17) is $(x, y)^{*}=(1,1)$.

We have used the piecewise linearization of objective function and constraint functions to approximate non-smooth programming problem (17) to a linear programming problem. The optimal solution of approximated linear programming problem for $n=10,20,50,100$ has been showed in Table 3.

Example 4: Consider following nonlinear nonsmooth programming problem:

$$
\begin{aligned}
& \text { Min } x^{2}+y^{2} \\
& \text { s.t } \\
& y+x^{2}-1 \leq 0 \\
& y-|x-1|^{3} \geq 0 \\
& 0 \leq x, y
\end{aligned}
$$

Table 3: The approximate optimal point of example 3

\begin{tabular}{|c|c|}
\hline $\mathrm{n}$ & Approximate optimal solution \\
\hline 10 & $(0.8103,1.172)$ \\
\hline 20 & $(0.9326,1.0740)$ \\
\hline 50 & $(0.9720,1.0337)$ \\
\hline 100 & $(0.9874,1.0025)$ \\
\hline
\end{tabular}

In this example, one of constraint functions is non-smooth function. The exact optimal solution of nonlinear programming problem $(18)$ is $(x, y)^{*}=$ $(0.35,0.27)$.

We have used the piecewise linearization of objective function and constraint functions to approximate non-smooth programming problem (18) to a linear programming (Figs. 4 and 5). The optimal solution of approximated linear programming problem for $n=10,20,50,100$ has been showed in Table 4.

\section{Conclusion}

We have solved the nonlinear smooth and nonsmooth programming problems by introducing a novel technique. Since, the competing methods in the literature need differentiability of objective and constraint functions, none of them is appropriate to solve the non-smooth problems. In this approach, we transfer the nonlinear programming problem to a variational problem. Then, we reduce it to a linear programming problem (LP). By solving the LP programming problem, we obtain the approximated solution of the original problem.

Table 4: The approximate optimal point of example 4

\begin{tabular}{|c|c|}
\hline $\mathrm{n}$ & Approximate optimal solution \\
\hline 10 & $(0.3188,0.2290)$ \\
\hline 20 & $(0.3385,0.2571)$ \\
\hline 50 & $(0.3413,0.2643)$ \\
\hline 100 & $(0.3482,0.2676)$ \\
\hline
\end{tabular}

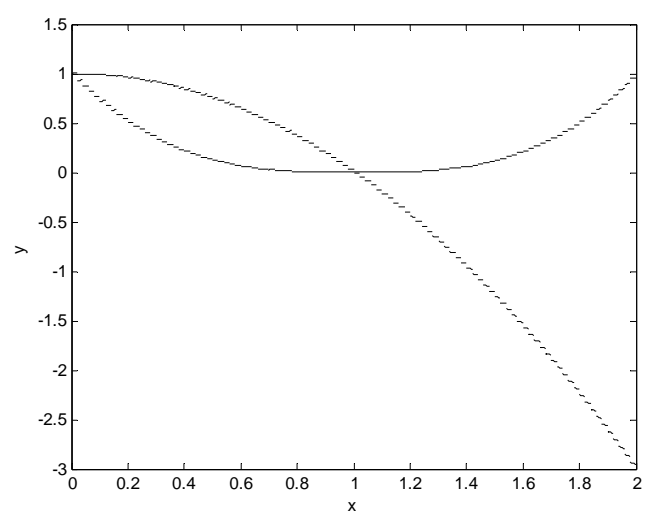

Fig. 4: The piecewise linearization of constraint functions of example $4(n=100)$

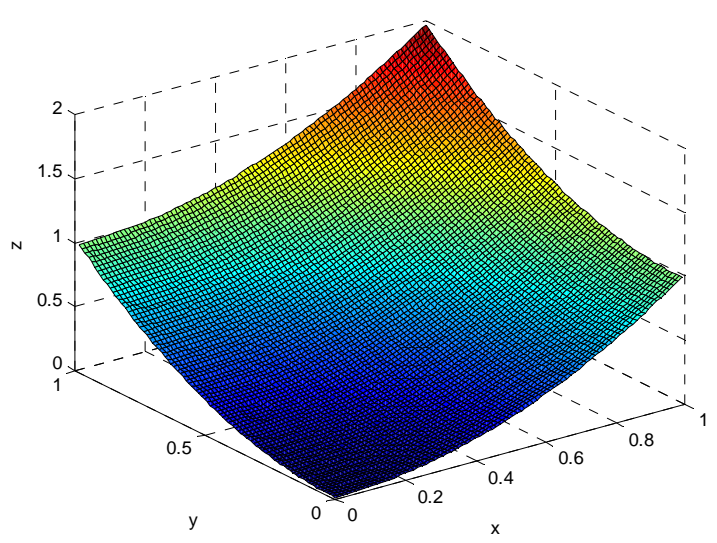

Fig. 5: The piecewise linearization of objective function of example $4(n=100)$.

\section{References}

Abhishek K, Leyffer S and Linderoth JT (2010). Modeling without categorical variables: a mixedinteger nonlinear program for the optimization of thermal insulation systems. Optimization and Engineering, 11(2): 185-212.

Bragalli C, D'Ambrosio C, Lee J, Lodi A and Toth P (2006). An MINLP solution method for a water network problem. In European Symposium on Algorithms, Springer Berlin Heidelberg, Lecture Notes in Computer Science, Berlin: Springer, 4168: 696-707 
Corsano G, Vecchietti AR and Montagna JM (2011). Optimal design for sustainable bioethanol supply chain considering detailed plant performance model. Computers and Chemical Engineering, 35(8): 1384-1398.

Griffith RE and Stewart RA (1961). A nonlinear programming technique for the optimization of continuous processing systems. Management Science, 7(4): 379-392.

Grossmann IE and Sargent RW (1979). Optimum design of multipurpose chemical plants. Industrial and Engineering Chemistry Process Design and Development, 18(2): 343-348.

Guerra A, Newman AM and Leyffer S (2011). Concrete structure design using mixed-integer nonlinear programming with complementarity constraints. SIAM Journal on Optimization, 21(3): 833-863.

Han SP (1976). Superlinearly convergent variable metric algorithms for general nonlinear programming problems. Mathematical Programming, 11(1): 263-282.

Han SP (1977). A globally convergent method for nonlinear programming. Journal of optimization theory and applications, 22(3): 297-309.

Harjunkoski I, Westerlund T and Pörn R (1999). Numerical and environmental considerations on a complex industrial mixed integer non-linear programming (MINLP) problem. Computers and Chemical Engineering, 23(10): 1545-1561.

Kamyad AV, Mehne HH and Borzabadi AH (2005). The best linear approximation for nonlinear systems. Applied Mathematics and Computation, 167(2): 1041-1061.

Misener R and Floudas CA (2012). Global optimization of mixed-integer quadraticallyconstrained quadratic programs (MIQCQP) through piecewise-linear and edge-concave relaxations. Mathematical Programming, 136(1): 155-182.

Murray W and Shanbhag UV (2006). A local relaxation approach for the siting of electrical substations. Computational Optimization and Applications, 33(1): 7-49.

Nesterov Y (2005). Smooth minimization of nonsmooth functions. Mathematical Programming, 103(1): 127-152.

Pardalos PM, Chaovalitwongse W, Iasemidis LD, Sackellares JC, Shiau DS, Carney PR and Yatsenko VA (2004). Seizure warning algorithm based on optimization and nonlinear dynamics. Mathematical Programming, 101(2): 365-385.

Rockafellar RT (1994). Nonsmooth optimization. Mathematical Programming: State of the Art, University of Michigan Press, Ann Arbor: 248258. 